\title{
Characterization and Evaluation of Porous Vermiculite Containing Polyethylene Composites Film
}

\author{
Hye Sun Lee and Jeong Ho Chang ${ }^{\dagger}$ \\ Center for Convergence Bioceramic Materials, Korea Institute of Ceramic Engineering and Technology, \\ Cheongju 28160, Korea
}

(Received November 16, 2017; Revised December 5, 2017; Accepted December 11, 2017)

\begin{abstract}
This work reported the preparation and evaluation of a freshness-keeping film prepared by composite of a porous ceramic material such as vermiculite and polyethylene polymer. The ceramic material was pretreated physically and chemically to control the specific surface areas and particle size. A high content master-batch was prepared using the pretreated vermiculite. The master-batch, which contained 30\% ceramic material, was mixed with a polymer material to prepare a film containing $3 \%$ vermiculite. The oxygen permeability and various physicochemical properties were evaluated for the prepared films. Compared to plain polyethylene film, the vermiculite loaded polyethylene film has a freshness maintenance property, indicating the creation of an improved film.
\end{abstract}

Key words : Vermiculite, Polyethylene, Films, Porous Ceramic, Composites

\section{Introduction}

E nhancing the quality of life and living standards of society is a dominant aspect of the highly industrialized modern society today. In this regard, functional packaging materials are applied in various areas of everyday life and are intimately related to the improvement of quality of life. Whereas packaging materials in the past were used to simply preserve the condition of a product, today's materials incorporate technology that benefits consumers through water vapor permeation control, product performance improvements, and prevention of microbial contamination, food spoilage, and component oxidation and discoloration. ${ }^{1-3)}$

In particular, functional packaging has the advantages of maintaining the properties of a product and extending product shelf life. Developments of films that provide such functionality as antioxidation, ${ }^{4)} \mathrm{UV}$ shielding, ${ }^{5)}$ heat resistance, ${ }^{6)}$ and antibiosis $^{7}$ are being pursued for application in various fields. Among these, film development methods for maintaining freshness, ${ }^{8)}$ including precooling that reduces respiration and low temperature storage like cold distribution, allow for long-term storage; however, films differ significantly depending on the appropriate composition ratio and type of each product category, and the costs of maintenance and equipment are high. Although it has been reported that antimicrobial formulae ${ }^{9,10)}$ to prevent contamination by bacteria and microorganisms show outstanding efficiency, there is the difficulty of determining the application concen-

\footnotetext{
${ }^{\dagger}$ Corresponding author: Jeong Ho Chang

E-mail : jhchang@kicet.re.kr

Tel : +82-43-913-1501
}

tration and dealing with the hazardousness of the antimicrobial content. Gas barrier technology, ${ }^{11-12)}$ used to selectively control the oxygen and carbon dioxide concentrations, allows the penetration of oxygen and carbon dioxide; this method requires caution as the gas composition within the film is sensitive to the surrounding temperature. Lastly, there is technology that enhances the freshness of products by controlling ethylene gas. The method of controlling ethylene gas absorption by mixing inorganic materials like clay and zeolite in the packaging material provides the advantages of convenient application to film in the manufacturing stage and relatively low cost compared to other techniques. Also, the method allows the loading and discharging of functional substances to porous materials; as such, research on functionality improvement continues today. ${ }^{13-16)}$

Vermiculite has characteristics similar to those of the inorganic ceramic materials such as clay and zeolite; as vermiculite is a hydrated magnesium, aluminum silicate mineral, its appearance is similar to that of mica. ${ }^{17,18)}$ Vermiculite is easily found in numerous countries worldwide and is conveniently decomposed in acid; purification and modification of ceramic materials using vermiculite are outstanding; this material is also chemically nontoxic, stable, and has significant cation exchange capacity. Thus, vermiculite is widely used in various fields including construction, industry, horticulture, and agriculture. ${ }^{19-21)}$ Since control of the pore size and reprocessing of conventional clay and zeolite are inconvenient, ${ }^{22)}$ vermiculite is a suitable material to overcome such disadvantages.

In this study, the properties of conventional polyethylene film fabrication material and inorganic material composite functional film were compared to investigate the effect of 
ceramic material addition. For the inorganic material, vermiculite was acid treated to fabricate a porous material with enhanced pore characteristics; then, the mixture ratio with polyethylene polymer was controlled to assess the physical properties of the film, while the effects of the ceramic material mixture on the functionality of the film, including oxygen and water permeability, were examined.

\section{Experimental Procedure}

The vermiculite and hydrochloric acid for the acid treatment process used in this study were manufactured by Sigma Aldrich; the low-density polyethylene (LDPE) was manufactured by Yuwon Chemicals.

Figure 1 provides a flowchart of the physical and chemical processing of vermiculite: the particles of the ceramic inorganic material vermiculite underwent planetary milling for $1 \mathrm{~h}$ in a clockwise direction, followed by no milling for 30 minutes, and then counterclockwise direction milling for 1 $\mathrm{h}$. The milling was carried out for a total of $2 \mathrm{~h}$ at $300 \mathrm{rpm}$. After the milling process, $10 \mathrm{~g}$ of vermiculite was added to $2 \mathrm{M} \mathrm{HCl}$; mixture was then stirred under reflux for $8 \mathrm{~h}$ at $120^{\circ} \mathrm{C}$. It was then cooled at room temperature and then cleaned multiple times using distilled water. After checking that there was no remaining $\mathrm{HCl}$ on the specimen, the specimen was acquired through a filter and through vacuum drying. As a result, porous vermiculite was produced. In order to investigate the material properties, a Micromeritics TriStar II-3020 specific surface area analyzer was used to measure the pore size and specific surface area based on the absorption and desorption results of nitrogen; SEM (SM300, Topcon) was used to observe the particle morphology. A particle size analyzer (LA950, Horiba, in water) was used to measure the particle size distribution; the vermiculite particle sizes before and after the physical and chemical pretreatment were measured.

A master batch is material fabricated by mixing together materials of intended functionality when polymer materials are to be processed as the main ingredient using methods of extrusion or injection. After the milling and acid treatment processes used in the experiment, the porous vermiculite and LDPE polymer were mixed at a ratio of $30: 70$ and dried in a dryer. Then, samples were moved with a hopper and discharged through an extruder to finally obtain a porous ceramic material mixed LDPE polymer master batch.

The master batch was polymer chips with a total ceramic content of $30 \%$. To obtain a thickness of $3 \mu \mathrm{m}$, the blown extrusion production method was used according to the mixture ratio between the master batch and polymer; then, a bag making machine was used to postprocess the material into bag form. Here, the film was fabricated with a ceramic content of $3 \%$; the haziness (model name: MURAKAMI HM150, light source: halogen lamp), oxygen permeability (model name: OX-TRAN Model 2/21, US Mocon), and water permeability (model name: Permatran-W 3/33 MA, US Mocon) were measured to investigate the effect of the ceramic material. The oxygen permeability and measurement range were $23 \pm 2^{\circ} \mathrm{C}$ and $0.05 \sim 10000\left(\mathrm{~cm}^{3} / \mathrm{m}^{2} \cdot 24 \mathrm{~h} \cdot \mathrm{atm}\right)$, respectively, and the water permeability test environment and measurement range were $38 \pm 2^{\circ} \mathrm{C}$ and $(100) \% \mathrm{R} . \mathrm{H}, 0.05 \sim 500(\mathrm{~g} /$ $\mathrm{m}^{2}$-day), respectively. The basis for the ceramic content in the film was the concentration at which the film retains its transparency and does not undergo discoloration due to the ceramic material. So, a concentration of $3 \%$ was selected. Moreover, additional research on the polymer composite ceramic content with optimal freshness maintenance functionality is necessary with regard to controlling the localized agglomeration of the polymer and identifying the issues of discoloration, property degradation, and cost increase, which can occur from mixing polymer at high concentrations.

Also, FE-SEM (model name: JEOL JSM-6700F), EDX (model name: Genesis 2000 XMS system), and TGA (model name: TA SDT Q600, measurement range: $\mathrm{RT} \sim 550^{\circ} \mathrm{C}$, nitrogen atmosphere) were used to observe the ceramic within the film, determine the presence of ceramic content within the film, and determine the ceramic content within the film, respectively.

\section{Results and Discussion}

Figure 2(a) shows SEM images identifying the porous vermiculite particle morphology after the milling and acid treatment processes. It was observed that the particle size of the initial vermiculite material was nonuniform, between $20-30 \mu \mathrm{m}$, while the particle size of the vermiculite after physical and chemical preprocessing was below $2 \mu \mathrm{m}$.

Figure 2(b) shows the SEM and particle size analysis results. The particle size of the initial vermiculite material was nonuniform, between $10-100 \mu \mathrm{m}$. Meanwhile, the particle size of the vermiculite after the physical and chemical preprocessing was below $1-4 \mu \mathrm{m}$. As the particle size became smaller, the agglomeration phenomenon intensi-

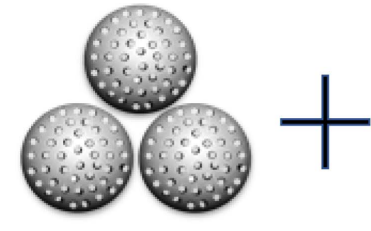

Porous Ceramic materials (Vermiculite)

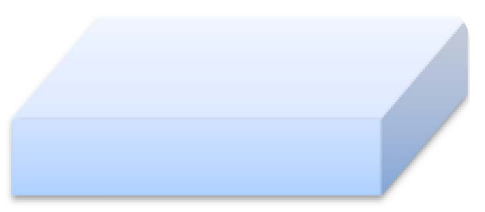

Polymer (Polyethylene)

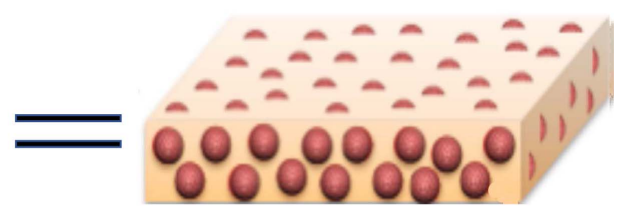

Functional packaging film (ceramic-polymer composite)

Fig. 1. Flowchart of vermiculite pretreatment process. 
(a)

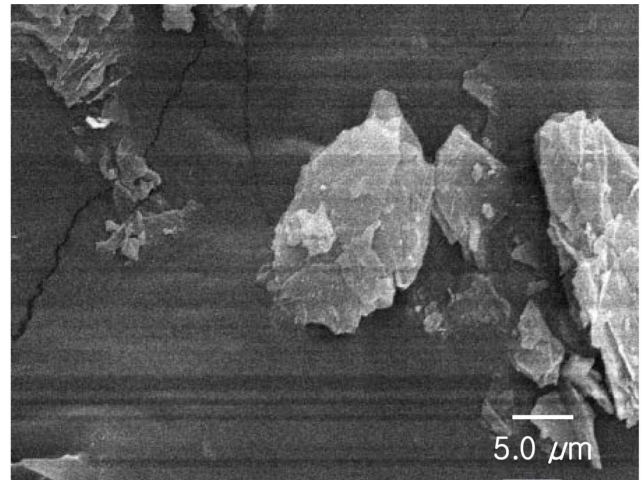

(b)

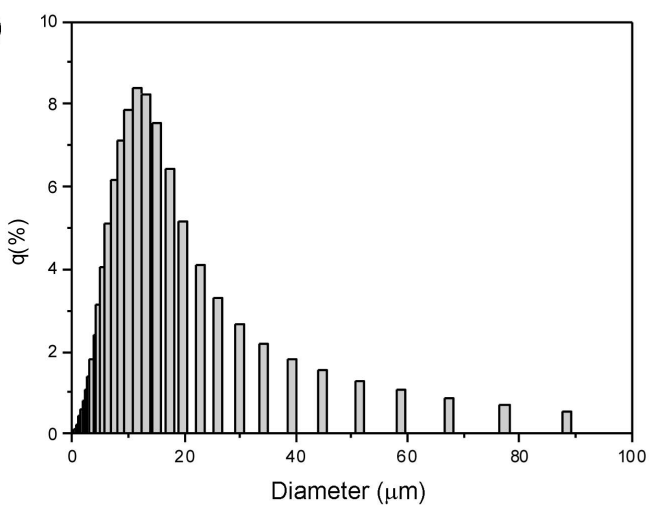

(c)

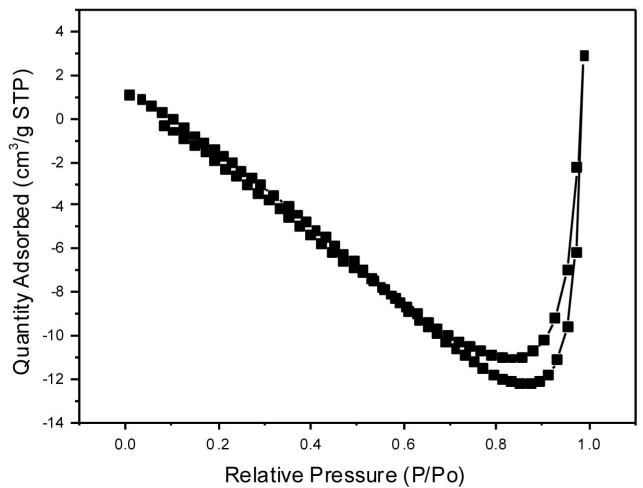

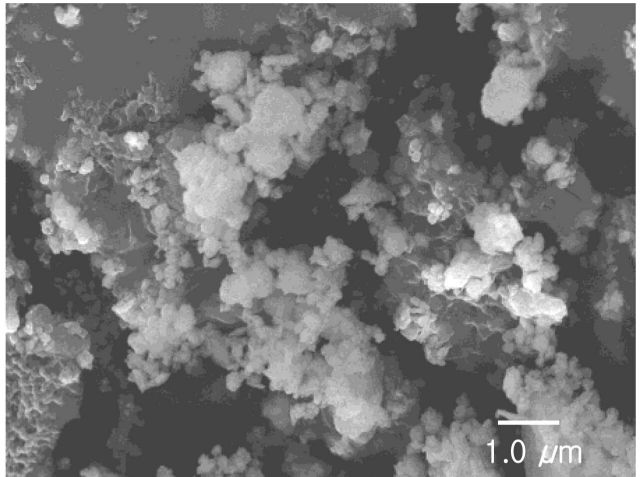
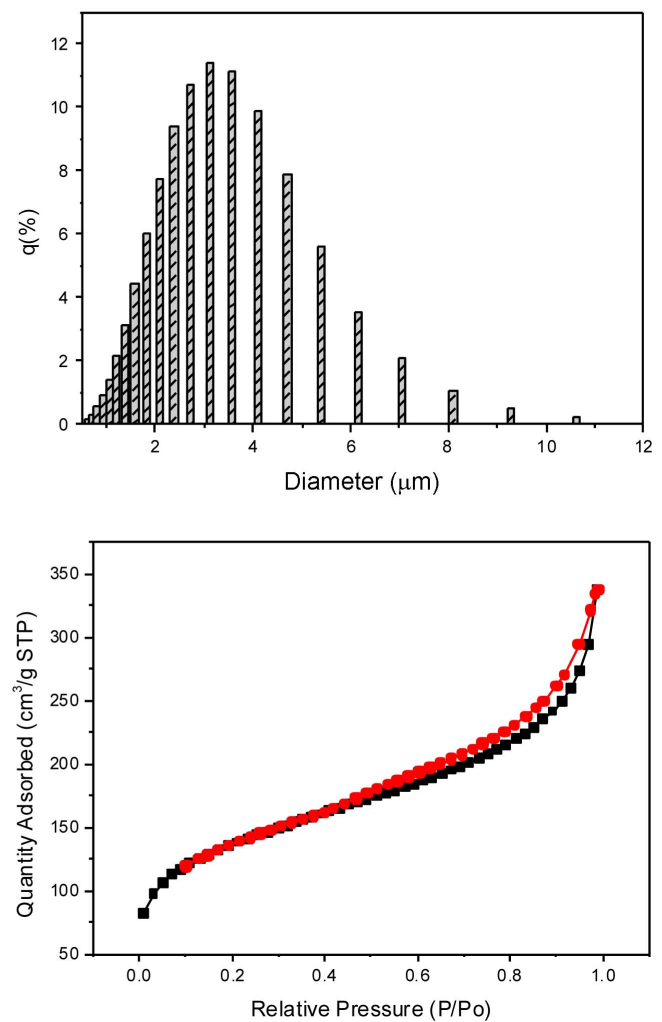

Fig. 2. (a) SEM images of vermiculites, (b) particle size distribution, and (c) their nitrogen adsorption-desorption isotherms before/after grinding.

fied, resulting in the particle size distribution appearing larger than the actual particle size. Fig. 2(c) shows the absorption and desorption isotherm, pore size, and specific surface area measurement results; these can be used to analyze the pore morphologies of porous materials. The initial vermiculite showed a low specific surface area of $5.8 \mathrm{~m}^{2} /$ $\mathrm{g}$ and low pore volume of $0.004 \mathrm{~cm}^{3} / \mathrm{g}$, while the average pore volume and specific surface area were $0.48 \mathrm{~cm}^{3} / \mathrm{g}$ and $464.2 \mathrm{~m}^{2} / \mathrm{g}$, respectively, for the material after the physical and chemical preprocessing. Based on these results, it was observed that the milling and acid treatment processes applied to the vermiculite produced a material with specific surface area improvement and particle size control.

Figure 3(a-b) provides FE-SEM image obtained to observe the distribution of the physically and chemically preprocessed vermiculite specimen within the film; Fig. 3(c) shows results of EDX measurement, conducted to determine that the particles within the film are ceramic materials. In the case of the polyethylene film that did not include ceramic material, only the polymer was observed, while the film with $3 \%$ ceramic material revealed that the ceramic material was spread out within the film. The EDX analysis identified the ceramic materials $\mathrm{Si}$ and polymer C.

Figure 4 shows the results of TGA, performed to determine the content of physically and chemically preprocessed ceramic material vermiculite within the film. The film with $0 \%$ ceramic material was found to have completely pyrolyzed at temperatures above $400^{\circ} \mathrm{C}$; the film from the mas- 
(a)

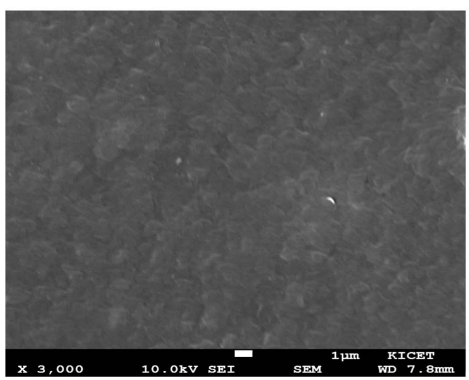

(b)

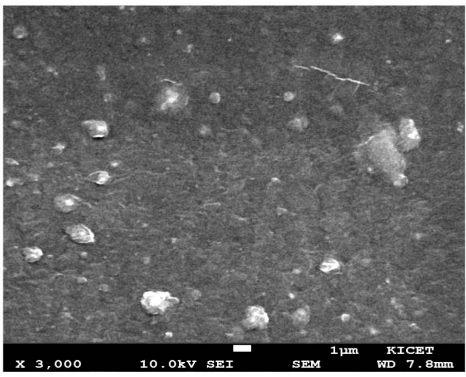

(c)

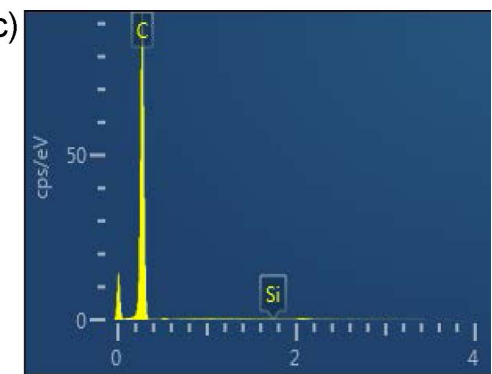

Fig. 3. FE-SEM images of vermiculite containing polyethylene films as a function of vermiculite concentration (a) $0 \%$, (b) $3 \%$, and (c) EDX analysis.

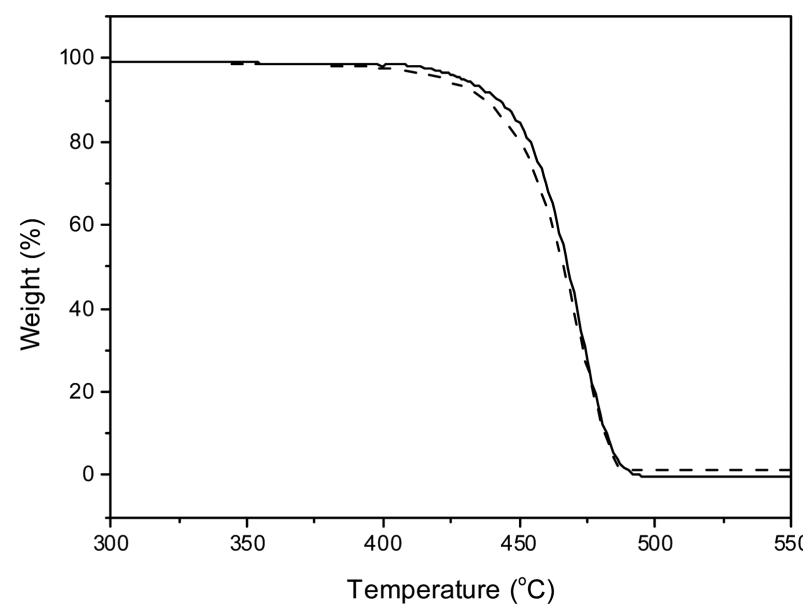

Fig. 4. TGA analysis of pre-processed vermiculite loaded Polyethylene film (0\%: solid, 3\%: Dash).

ter batch with $3 \%$ ceramic material actually had a ceramic material content of approximately $1.34 \%$. This result shows a lower ceramic material content than that of the initially included ceramic material due to loss that occurs during the ceramic material and polymer mixing process. Based on these experimental results, it was determined that the distribution of physically and chemically preprocessed $3 \%$ content vermiculite within the film was excellent and mixing with polyethylene polymer was also observed. The haziness, oxygen permeability, and water permeability of the conventional polyethylene film and the polyethylene film with vermiculite of $3 \%$ content fabricated under the same conditions were measured to evaluate film properties.

Table 1 shows the determined film properties. The haziness represented the transmittance of light through the sample. Since observation of the product interior is more convenient for films with high transparency, product appli- cation of hazy films can be difficult.

$$
\begin{array}{r}
\text { Haze }=\text { DF } / T T \times 100 \text { (DF: Diffusion transmittance, } \\
\text { TT: Total light transmittance) }
\end{array}
$$

The measurement results revealed a relatively high haziness of the ceramic material with added polyethylene film compared to the regular polyethylene film; however, the haziness was not so severe as to make identification of the product inside the film impossible. The oxygen and water permeabilities were measured using ASTM D3985 and ASTM F1249 standards. The oxygen and water permeabilities of the preprocessed vermiculite-included film were 8570 $\mathrm{cm}^{3} / \mathrm{m}^{2} \cdot 24 \mathrm{~h} \cdot$ atm and $18.16 \mathrm{~g} / \mathrm{m}^{2}$ day, respectively; those permeabilities of the regular film were $15.6 \%$ and $13.3 \%$ of those values, respectively. These good results, compared to those for the polymer material, are due to the flow of air through the porous ceramic particles; the influence of the ceramic material content needs to be studied further.

The results of this study reveal that the application of ceramic material with outstanding pore characteristics resulted in film property enhancement.

\section{Conclusions}

In this study, the mineral vermiculite was used to enhance the specific surface area and pore volume through milling and acid treatment processes; this was followed by hybridization with low density polyethylene to compare the properties of this new film, according to inorganic ceramic material content, with regular polyethylene film. The physically and chemically preprocessed ceramic material vermiculite had an average particle size below $1 \mu \mathrm{m}$, specific surface area of $464.20 \mathrm{~m}^{2} / \mathrm{g}$, and pore volume of $0.48 \mathrm{~cm}^{3} / \mathrm{g}$. Compared to the initial material, the particle size decreased around 20 times, the specific surface area increased around

Table 1. Haze, Oxygen Permeability and Water Permeability Results for Polyethylene Film and 3\% Vermiculite Loaded Polyethylene Film

\begin{tabular}{cccc}
\hline $\begin{array}{c}\text { Vermiculite content } \\
(\%)\end{array}$ & $\begin{array}{c}\text { Haze } \\
(\%)\end{array}$ & $\begin{array}{c}\text { Oxygen permeability } \\
\left(\mathrm{cm}^{3} / \mathrm{m}^{2} \cdot 24 \mathrm{~h} \cdot \mathrm{atm},(23 \pm 2){ }^{\circ} \mathrm{C}\right)\end{array}$ & $\begin{array}{c}\text { Water permeability } \\
\left(\mathrm{g} / \mathrm{m}^{2} \cdot \mathrm{day},(38 \pm 1){ }^{\circ} \mathrm{C}, 100 \% \mathrm{R} . \mathrm{H}\right)\end{array}$ \\
\hline 0 (control) & 7.80 & 7229 & 15.74 \\
3 & 20.94 & 8570 & 18.16 \\
\hline
\end{tabular}


80 fold, and the pore volume increased 120 fold. The physically and chemically preprocessed material was fabricated using a $30 \%$ high content master batch; a ceramic-polymer film with $3 \%$ polyethylene polymer film and ceramic material was fabricated. The dependence of the properties on the ceramic content and the changes of the film properties was observed. The haziness, oxygen permeability, and water permeability were measured for the ceramic-polymer film containing 3\% ceramic material and for the conventional nontreated polyethylene film. The haziness increased due to the included ceramic material, but the oxygen and water permeabilities increased by $15.6 \%$ and $13.3 \%$, respectively, compared to those values of the regular polyethylene film. In this study, ceramic material was found to have an effect on the film properties and it was determined that application to fruits and vegetables would be effective since these products are significantly affected by moisture and oxygen.

\section{Acknowledgments}

This research was supported by the Ministry of Trade, Industry and Energy (MOTIE, Korea), part of the Industry Core Technology Development Program funded by the Korea Evaluation Institute of Industrial Technology (KEIT) [10063291].

\section{REFERENCES}

1. S. W. Kim, "Functional Polymer Packing Materials (in Korean)," Polym. Sci. Technol., 12 [2] 163 (2001).

2. H. K. Han and C. Y. Park, "Functional food Packaging Film (in Korean)," Polym. Sci. Technol., 12 [2] 174-82 (2001).

3. S. Tamarindo and C. Pastore, "Packaging Film Impact on Food Organoleptic Properties: An Experimental Study," J. Appl. Packag. Res., 8 [4] 78-87 (2015).

4. Y. J. Byun, Y. T. Kim, and S. Whiteside, "Characterization of an Antioxidant Polylactic Acid (PLA) Film Prepared with a-tocopherol, BHT and Polyethylene Glycol Using Film Cast Extruder," J. Food Eng., 100 [2] 239-44 (2010).

5. M. K. Hedayati, A. U. Zillohu, T. Strunskus, F. Faupel, and M. Elbahri, "Plasmonic Tunable Metamaterial Absorber as Ultraviolet Protection Film," Appl. Phys. Lett., 104 [041103] 1-5 (2014).

6. T. Ebina and F. Mizukami, "Flexible Transparent Clay Films with Heat-Resistant and High Gas-Barrier Properties," Adv. Mater., 19 [18] 2450-53 (2007).

7. B. Malhotra, A. Keshwani, and H. Kharkwal, "Antimicrobial Food Packaging: Potential and Pitfalls," Front Microbiol., 6 [611] 1-9 (2015).

8. A. G. Perez, R. Olias, J. M. Olias, and C. Sanz, "Strawberry Quality as a Function of the "High Pressure Fast Cooling" Design," Food Chem., 62 [2] 161-68 (1998).

9. H. S. Lee and J. H. Chang, "Antimicrobial Ceramic Hybrid Polyethylene Film with Chamomile Extracts for Feed
Packaging," J. Korean Ceram. Soc. 54 [3] 228-34 (2017).

10. G. S. Kyung and S. H. Ko, "A Study of Antibacterial Paper Packaging Materials Coated with Chitosan-Ag Nanocomposite Prepared by Green Synthesis (in Korean)," J. Kor. TAPPI, 46 [2] 8-15 (2014).

11. F. R. Harker, H. J. Elgar, C. B. Watkins, P. J. Jackson, and I. C. Hallett, "Physical and Mechanical Changes in Strawberry Fruit after High Carbon Dioxide Treatments," Postharvest Biol. Technol., 19 [2] 139-46 (2000).

12. C. L. Moretti, L. M. Mattos, A. G. Calbo, and S. A. Sargent, "Climate Changes and Potential Impacts on Postharvest Quality of Fruit and Vegetable Crops: A Review," Food Res. Int., 43 [7] 1824-32 (2010).

13. M. S. Aday and C. Caner, "The Shelf Life Extension of Fresh Strawberries Using an Oxygen Absorber in the Biobased Package," LWT-Food Sci. Technol., 52 [2] 102-9 (2013).

14. G. O. Lim, S. A. Jang, H. Y. Kim, H. J. Kim, and K. B. Song, "Use of a Gelatin Film Containg Grapefruit Seed Extract in the Packaging of Strawberry (in Korean)," Korean J. Food Preserv., 17 [2] 196-201 (2010).

15. A. A. Kader and S. Ben-Wehoshua, "Effects of Super Atmospheric Oxygen Levels on Postharvest Physiology and Quality of Fresh Fruits and Vegetables," Postharvest Biol. Technol., 20 [1] 1-13 (2000).

16. Korea Food Research Institute, "Development of New Functional MA Packaging Methods for Freshness Extension of Agricultural Produces," 1998.

17. K. K. Syrmanova, M. T. Suleimenova, A. Y. Kovaleva, N. Y. Botabayev, and Z. B. Kaldybekova, "Vermiculite Absorption Capacity Increasing by Acid Activation," Orient. J. Chem., 33 [1] 509-13 (2017).

18. J. Keay and A. Wild, "The Kinetics of Cation Exchange in Vermiculite," Soil Sci., 92 [1] 54-60 (1961).

19. T. T. Li, Y. C. Chuang, C. H. Huang, C. W. Lou, and J. H. Lin, "Applying Vermiculite and Perlite Fillers to SoundAbsorbing/Thermal-Insulating Resilient PU Foam Composites," Fibers Polym., 16 [3] 691-98 (2015).

20. I. G. Svegl, B. Ogorevc, and V. Hudnik, "A Methodological Approach to the Application of a Vermiculite Modified Carbon Paste Electrode in Interaction Studies: Influence of Some Pesticides on the Uptake of $\mathrm{Cu}(\mathrm{II})$ from a Solution to the Solid Phase," Anal. Bioanal. Chem., 354 [5-6] 770-73 (1996).

21. S. Fujimura, K. Yoshioka, T. Saito, M. Sato, M. Sato, Y. Sakuma, and Y. Muramatsu, "Effects of Applying Potassium, Zeolite and Vermiculite on the Radiocesium Uptake by Rice Plants Grown in Paddy Field Soils Collected from Fukushima Prefecture," Plant Prod. Sci., 16 [2] 166-70 (2013).

22. U. Olsbye, S. Svelle, M. Bjorgen, P. Beato, T. V. W. Janssens, F. Joensen, S. Bordiga, and K. P. Lillerud "Conversion of Methanol to Hydrocarbons: How Zeolite Cavity and Pore Size Controls Product Selectivity," Ang. Chem., 51 [24] 5810-31 (2012). 